\title{
Elecciones de 2012 en Estados Unidos. Peso del voto «latino" y perspectivas de reforma a la politica de inmigración
}

\author{
Oscar Chacón
}

$\mathrm{E}$ 1 pasado 6 de noviembre de 2012 concluyó la contienda electoral en Estados Unidos. El presidente Barack Obama aseguró su reelección para un segundo cuatrienio. Como es sabido, la elección presidencial en Estados Unidos no se decide por medio del voto directo, sino por medio de un sistema de voto indirecto conocido como Colegio Electoral. El número total de votos colegiados es igual a la suma de los distritos congresionales que existen en el país (438), más el número total de puestos senatoriales federales (100). Por lo tanto, para poder ganar la elección presidencial, un candidato debe ganar cuando menos 270 votos del Colegio Electoral.

El presidente Obama aseguró su reelección con 332 votos del Colegio Electoral, mientras que el candidato republicano, Mitt Romney, contó 
sólo con 206. Obama obtuvo un total de $62,611,250$ votos versus $59,134,475$ votos obtenidos por Romney. La diferencia en número de votos entre el ganador y el perdedor fue de 3.5 millones, aproximadamente. A pesar de que la diferencia, en lo que se refiere al voto popular, es relativamente pequeña entre ambos candidatos, la amplia diferencia en el número de votos del Colegio Electoral obedece a la lógica de «el ganador se lleva todo». Debido a la forma en que opera el sistema, en ocasiones anteriores ha sido posible que el ganador de la elección, según los votos colegiados, no lo haya sido según el voto popular.

En el ámbito del voto popular, es importante destacar que hubo un decrecimiento en la participación electoral este año. De acuerdo con datos preliminares, la tasa de participación electoral fue de 57.5\%. Para efectos comparativos, la tasa de participación electoral en 2008 fue de $62.3 \%$ y, en 2004 , de $60.4 \%$. De acuerdo con el Pew Hispanic Center, los votantes potenciales en este año eran, aproximadamente, 215 millones de personas, inclu- yendo 23.7 millones de personas de origen latinoamericano. De acuerdo con la edición del semanario The Economist del 10 de noviembre, el presidente Obama contó con 50.4\% del voto popular, comparado con 48\% obtenido por el candidato republicano. A partir de lo reportado por este medio, $1.6 \%$ del voto popular fue para candidatos de terceros partidos.

Además de las elecciones presidenciales, se llevaron a cabo elecciones legislativas federales que involucraron a cada uno de los miembros de la Casa de Representante, como también a un tercio de los 100 miembros del Senado. En términos generales, los resultados de estas elecciones no produjeron un cambio sustantivo ni en la composición numérica ni en la orientación política de cada una de las cámaras legislativas federales. Sin embargo, a pesar de que la correlación numérica de votos en ambas cámaras legislativas, entre demócratas y republicanos, seguirá siendo virtualmente la misma en el próximo Congreso, la iniciativa política estará en manos del Partido Demócrata, debido a que el 
liderazgo de la bancada republicana en ambas cámaras declaró a principios de la primera administración de Obama que su más importante meta iba a ser asegurar que el presidente no fuese reelecto.

En el caso del Senado, el Partido Demócrata contará con 53 senadores; además, fue electo un candidato independiente del estado de Maine, lo cual eleva el número de senadores independientes a dos. Estos dos senadores se adherirán, seguramente, al bloque de legisladores demócratas a la hora de ejercer su voto, lo cual conllevará a una ventaja tácita de 55 senadores para el lado demócrata. El Partido Republicano contará con 45 senadores. Senadores republicanos conocidos por su inclinación bipartidista, como Olympia Snow, de Maine, Scott Brown, de Massachusetts, y Richard Lugar, de Indiana, ya no estarán en el Senado. En principio, este cambio hace que la idea del bipartidismo como nueva manera de funcionar entre ambos partidos no sea algo previsible.

En la Casa de Representantes —o Cámara Baja一, el Partido Re- publicano logró mantener su condición de mayoría: contará con 234 miembros, mientras que el Partido Demócrata lo hará con 201 miembros. La orientación política de ambas bancadas será muy parecida a la que ha estado vigente hasta ahora, de forma similar a lo sucedido en el Senado.

Independientemente del balance de fuerzas de cada una de las bancadas legislativas federales, el presidente Obama y el Partido Demócrata llegarán a la próxima sesión legislativa con la iniciativa política a su favor. Si el presidente Obama quisiera apuntar hacia un legado estratégico para la nación, su victoria electoral le da la posibilidad de responder de una manera más aguda, genuinamente nueva ante los grandes desafíos de la nación. Desde la administración Reagan, el Partido Republicano ha ejercido una hegemonía política e ideológica que sigue vigente hasta hoy. E1 presidente Obama tiene la posibilidad de inyectar un nuevo rumbo al país que le permita a Estados Unidos resolver positivamente $-\mathrm{y}$ desde un planteamiento mucho 
más inclusivo y sostenible a largo plazo - temas trascendentales para la nación, como la orientación general de la política económica, la política fiscal, la política exterior, el papel del Estado como ente regulador y garante del bienestar social, la política ecológica, la política de inmigración, el mantenimiento y el desarrollo infraestructural, la política de educación pública, una política universal de acceso a la salud, etcétera. Si prevalece la actitud política que ha caracterizado al Partido Demócrata desde finales de la década de los setenta, es decir, la de una conducta de sometimiento a la hegemonía republicana, se echará a perder, una vez más, la oportunidad de un momento histórico favorable para cambios trascendentales.

\section{El papel de los votantes DE ORIGEN LATINOAMERICANO}

Aunque los datos hasta ahora conocidos son todavía preliminares, se estima que entre 11 y 12.5 millones de personas de origen latinoamericano votaron en las pasadas eleccio- nes. Esta cifra significa que el voto latinoamericano se incrementó en 2.7 millones de nuevos votantes, con relación al número de votos emitidos en 2008. De acuerdo con los datos preliminares, $71 \%$ de los votantes de origen latinoamericano apoyó la reelección del presidente Obama; siguiendo el patrón de apoyo del electorado en general, las mujeres de origen latinoamericano fueron su principal apoyo, al otorgar $77 \%$ de su votación; por su parte, los hombres de origen latinoamericano otorgaron $65 \%$ de sus votos. Este grado de preferencia electoral superó el apoyo recibido por Barack Obama en las elecciones de 2008, cuando consiguió $67 \%$ del voto de personas de origen latinoamericano, el cual fue especialmente benéfico para su reelección en los estados clave para el aseguramiento de la mayoría de los votos del Colegio Electoral, tales como Ohio, Virginia, Wisconsin, Iowa, Colorado y Nevada. En el caso de Florida, el papel de este segmento de votantes fue también de mucha importancia, aunque en esta ocasión la elección presidencial se definió aun antes de 
que el escrutinio oficial de votos en Florida hubiera concluido.

De acuerdo con lo reportado por la revista BusinessWeek en su edición del 12 de noviembre, el grado de apoyo recibido por Obama de los votantes de origen latinoamericano fue consistente con el grado de apoyo recibido por otros grupos minoritarios del electorado estadounidense. La comunidad negra le otorgó $93 \%$ de su voto, mientras que las personas de origen asiático $73 \%$. El flanco débil de Obama fue el del grupo de votantes de raza blanca: sólo sufragó $56 \%$ a favor. En cambio, $89 \%$ de los votos que recibió Mitt Romney provinieron de este grupo.

El grado de apoyo recibido por el presidente Obama y los candidatos del Partido Demócrata para la Casa de Representantes y del Senado por los votantes de origen latinoamericano deberá ser objeto de mayor escrutinio en el futuro. Sin embargo, parece seguro afirmar que la explicación más obvia de este abrumador apoyo en la elección del pasado 6 de noviembre se reduce a la percepción clara de que la opción republicana implicaría un empeoramiento de las condiciones generales de vida de esta comunidad en Estados Unidos.

Esta afirmación preliminar se basa en el siguiente hecho: desde la perspectiva de los estándares económico-sociales, la comunidad de origen latinoamericano ha experimentado un retroceso significativo a lo largo de los últimos años, particularmente por la epidemia de embargos hipotecarios, empeorada por la recesión económica que ha afectado a Estados Unidos a lo largo de los últimos años. De acuerdo con un estudio publicado por el Pew Research Center en julio de 2011, las personas de origen latinoamericano fueron las que experimentaron el mayor grado de pérdida de riqueza - 66\%- entre 2005 y 2009, comparado con otros grupos étnico-culturales. En el ámbito del empleo, los trabajadores de origen latinoamericano han sufrido mucho desde 2007, debido a la eliminación de puestos de trabajo en áreas en las que tiende a concentrarse el trabajador de origen latinoamericano, en especial en la industria de la construcción, que suele pagar salarios por encima de los devengados en la industria de servicios. 
En el plano más amplio del mercado laboral, si bien ha habido una recuperación en el empleo de población de origen latinoamericano, comparada con los agudos niveles de desempleo que se vivieran a lo largo de 2008 y 2009, también es cierto que los trabajadores de origen latinoamericano se han visto desproporcionalmente afectados por la tendencia general hacia un mayor encarecimiento del empleo y, por extensión, de las condiciones de vida. Esta tendencia se expresa en tasas salariales muy bajas, carencia de acceso a beneficios relacionados con el empleo —cuidado médico y vacaciones-y reemplazo sistemático de puestos de trabajo, que en otros tiempos tendían a ser de tiempo completo y que se han venido convirtiendo en puestos de medio tiempo, obtenidos, con frecuencia, por medio de agencias de empleo temporal. Esta tendencia ha sido una de las características de la «recuperación» que ha experimentado la economía estadounidense en lo referente al empleo a lo largo de los últimos años.

En el campo de la política de inmigración, los pasados cuatro años han sido muy duros para la comunidad inmigrante mexicana, centroamericana y latinoamericana, en general. La administración Obama se ha adjudicado la tristemente célebre condición del gobierno que más extranjeros ha deportado en la historia: cerca de un millón y medio de personas extranjeras han sido deportadas desde que Obama asumió la presidencia. Ha sido enorme el costo humano de esta práctica para millones de familias migrantes: se trata de millones de personas que han visto a sus familias separadas de forma forzosa, incluyendo cientos de miles de ciudadanos estadounidenses. Más allá de la población extranjera que reside en Estados Unidos sin autorización migratoria, la situación en torno a la población de residentes permanentes no ha sido placentera tampoco. Los costos de las solicitudes de residencia per- 1 manente, así como los de las solicitudes de naturalización han seguido incrementándose, y los periodos de espera para alcanzar la resolución plena de solicitudes de beneficios migratorios siguen siendo extremadamente largos. 
Además, la administración Obama ha promovido de manera sistemática un programa denominado «Comunidades Seguras» («S-Comm»), el cual requiere la cooperación de los cuerpos locales de policía con la policía federal migratoria conocida como ICE, por sus siglas en inglés. En un primer momento, la administración Obama afirmó que la cooperación de la policía local sería voluntaria. A la luz de cuestionamientos morales y políticos por los gobernadores de estados como Nueva York, Illinois y Massachusetts, que expresaron su deseo de poner fin a la colaboración con el gobierno federal en torno al programa S-Comm, la administración Obama respondió con la aclaración de que la cooperación en el marco de este programa era obligatoria y no voluntaria. S-Comm ha sido el principal mecanismo para la aprehensión, detención y eventual deportación de cientos de miles de extranjeros que residen en Estados Unidos sin autorización migratoria, la vasta mayoría de ellos sin haber cometido ningún tipo de acción criminal.
Sumado a lo anterior, estaba también la negativa de la administración Obama, hasta el verano de 2011, a utilizar la autoridad ejecutiva o presidencial para efectos de otorgar alivio migratorio a segmentos de la población extranjera que reside en el país sin autorización migratoria. El presidente Obama afirmó en repetidas ocasiones que él no tenía autoridad de otorgar alivio migratorio, a pesar de que el antecedente histórico demuestra que tanto presidentes republicanos como demócratas han hecho uso de dicha autoridad. Después de mucha presión política ejercida por las organizaciones a favor de los derechos de los inmigrantes $y$, en particular, por una ola de protestas hechas ante las oficinas de campaña en busca de su reelección, la Casa Blanca anunció, en agosto de 2011, el primero de varios programas de alivio administrativo para personas migrantes sin papeles. Desdichadamente, los programas administrativos otorgados fueron en última instancia ineficaces o muy lentos en su implementación. Su resultado neto ha sido muy inferior a la expectativa generada. 
Ejemplo de lo anterior fue el anuncio hecho a efecto de proveer alivio migratorio para cerca de 300 mil personas que en aquel entonces enfrentaban órdenes de deportación. La administración Obama anunció que serían procesados sólo los casos de personas con antecedentes criminales, insinuando que la mayoría de ellos serían dejados en libertad. Un año más tarde, el periódico New York Times reveló que el número total de beneficiarios de ese programa no llegó ni a diez mil personas.

Aun en el caso del programa de alivio migratorio para jóvenes migrantes sin papeles, anunciado el 15 de junio de este año, en plena campaña electoral y en un momento donde reinaba mucho escepticismo dentro de la comunidad latina en cuanto al apoyo que merecía el presidente Obama en camino a su reelección, el programa no arrancó sino a partir del 15 de agosto, y las tasas iniciales de aprobación eran muy bajas. Aunque se proyectó un universo de hasta 1.7 millones de beneficiarios, los números de solicitudes recibidas y aprobadas siguen muy distantes de esa cifra.
Todo lo anterior evidencia que el abrumador apoyo a favor de la reelección de Obama y en pro de los candidatos del Partido Demócrata ha tenido mucho más que ver con la percepción de los votantes de origen latinoamericano de que su situación sería mucho peor con una administración republicana, que con el grado genuino de satisfacción y entusiasmo generado por el desempeño de la administración Obama en relación con sus campos de interés directo.

Independientemente de las razones que llevaron a este grupo de votantes a apoyar al Partido Demócrata y a Barack Obama, el hecho es que la elección recién pasada ha marcado el inicio decisivo de un proceso irreversible: la diversificación del electorado estadounidense en el futuro. No se trata de que los datos actuales sean abrumadores en este sentido. A pesar del grado de atención dada al rol del «voto latino» en las elecciones recientes, los votantes de raza blanca representaron $72 \%$ del total de los votos emitidos en esta elección. Sin embargo, el futuro estará marcado por una 
tendencia continua de crecimiento de los votantes de minorías étnicas y raciales, encabezados por los votantes de origen latinoamericano, y por el decrecimiento sistemático en la proporción de votantes de raza blanca. La elección recién pasada ha dejado bastante bien evidenciado que el Partido Republicano, dada su agenda económica, social y política actual, está destinado a convertirse en una fuerza política cada vez más insignificante, a menos que haya un giro fundamental en el rumbo en que se han venido desplazando a lo largo de las últimas décadas.

\section{El Voto latino SObRe}

\section{LA REFORMA MIGRATORIA}

El factor que más atención ha generado desde la noche del 6 de noviembre ha sido la incapacidad del Partido Republicano para atraer significativamente a los votantes de minorías étnico-culturales, principalmente los votantes de origen latinoamericano. Aunque el desafío para el Partido Republicano es mucho más complejo, la prensa y la mayoría de los analistas políticos han adjudicado la incapacidad del Partido Republicano de atraer al «voto latino» debido a su posición sobre los derechos de los inmigrantes y la política de inmigración.

Es impresionante cómo los medios de prensa de habla inglesa, desde fuentes conservadoras, como la cadena Fox, hasta medios considerados liberales, como el periódico New York Times, han venido señalando de manera insistente la urgencia de que el Partido Republicano reevalúe su posición sobre los derechos de los inmigrantes y sobre la política de inmigración. Esta corriente de opinión ha conllevado el fuerte resurgimiento del debate público en torno a la necesidad de reformar la política de inmigración de Estados Unidos. El debate público se ha enfocado más en el caso del Partido Republicano, dando por sentado que el tema de la reforma a la política de inmigración no es igualmente relevante para el Partido Demócrata.

Señalar al Partido Republicano como la entidad política que está en el banquillo de los acusados en lo 
referente a política de inmigración, ignora el hecho de que la administración Obama y el Partido Demócrata han infligido un enorme costo social a las familias inmigrantes a lo largo de las últimas dos décadas. Más allá del hecho de que la administración Obama ha pasado ya a la historia como la administración federal que más personas extranjeras ha deportado, hay que recordar también que la ley de inmigración más punitiva de la historia contemporánea fue aprobada en 1996, durante la administración Clinton.

Si el énfasis generado en el ámbito del debate público nacional a partir de las elecciones recién pasadas en torno a la política de inmigración fuese más riguroso, se tendría que hablar de la necesidad de reconsiderar la lógica que sobre este tema ha predominado en Estados Unidos desde, cuando menos, los principios de los años noventa, independientemente del partido que ha estado en el control de la rama ejecutiva o legislativa del gobierno federal. Desdichadamente, la rigurosidad investigativa de la prensa estadunidense deja mucho que desear. Consecuentemente, la naturaleza del debate público sobre estos temas tiende a quedarse en un plano superficial y simplista y tiende a reducir el problema a un posible cambio de actitud del Partido Republicano.

Más allá de cuán profundo y riguroso ha sido el debate público reciente sobre la política de inmigración y el trato que se da a la población extranjera que vive en Estados Unidos, no hay duda de que estos temas serán importantes en la agenda política y legislativa de los próximos años. La reanudación de la promesa del presidente Obama de retomarlos durante su segundo término presidencial, combinado con las declaraciones hechas por líderes del ala más pragmática del Partido Republicano, parecen confirmar que se les pondrá mucha atención en los próximos años.

Con el fin de tratar de sacar el máximo provecho del reanudado interés en el tema de la política de inmigración, así como en lo referente a los derechos de los inmigrantes, se vuelve urgente redefinir la agenda política de incidencia, especialmente 
desde la perspectiva de las comunidades migrantes mismas.

Desde la perspectiva de la Alianza Nacional de Comunidades Inmigrantes Caribeñas y Latino Americanas (NALACC, por sus siglas en inglés), consideramos urgente ir mucho más allá del marco de reforma legislativa que ha predominado desde 2002, aproximadamente. Se trata del planteamiento político y legislativo conocido como Reforma Compresiva de Inmigración (CIR, por sus siglas en inglés). Este marco surgió a principios de la década pasada como una respuesta pragmática que aceptaba de manera tácita el marco punitivo establecido por la ley de inmigración de 1996, y buscaba situar el concepto de reforma dentro del marco político y legislativo establecido por dicha ley.

Desde la perspectiva de los liderazgos migrantes latinoamericanos, consideramos que para hablar de una agenda genuina de reforma a la política de inmigración actual, capaz de equipar al país con una política de inmigración funcional, humanista y de larga duración, es imperativo trascender la lógica que ha caracterizado el esquema denominado cIR. Consideramos que, si bien hay que partir de una visión integral y de largo plazo del tipo de reforma a la política de inmigración que se busca, debemos aprender de las lecciones de los últimos diez años y estar muy abiertos a la posibilidad de que la manera de avanzar hacia la meta última de contar con una ley de inmigración integralmente diferente a la actual, no será algo a lo que podamos llegar de un solo golpe, sino por medio de cambios graduales que a la larga nos lleven a la condición ideal. Consideramos también que, mientras el Congreso toma acción efectiva en el ámbito de reforma legislativa, la rama ejecutiva del gobierno debe seguir profundizando el uso de su poder para brindar alivio migratorio efectivo a poblaciones migrantes en condiciones especialmente meritorias. Un tema sobre el cual debemos todos aunar esfuerzos es el de la urgencia de parar el ritmo actual de deportaciones. La administración Obama debe ser forzada a cambiar su rumbo sobre este tema. 
¿QUÉ SE PUEDE ESPERAR?

Independientemente de cómo definamos la agenda de reforma a la política de inmigración, entre las comunidades migrantes organizadas y quienes nos apoyan, es importante tener muy claro el hecho de que, desde la perspectiva republicana, buscarán hacer lo menos posible y tratar de sacar el máximo beneficio publicitario de lo poco que se haga. Además, es muy importante estar claros en que el punto máximo de convergencia entre la dirigencia del Partido Demócrata y el ala más pragmática del Partido Republicano, en lo que se refiere a la política de inmigración, gira en torno a la idea de legalizar el acceso al trabajo. Esto quiere decir que el tipo de reforma sobre la cual se puedan prever posibilidades de colaboración entre ambos partidos seguramente será una cuya piedra angular sería un programa de empleo temporal para trabajadores extranjeros, sin una opción real hacia la condición de «residencia permanente», mucho menos hacia la ciudadanía.
Al evaluar las propuestas de reforma a la política de inmigración que han sido presentadas a lo largo de los últimos cinco años, es obvio que han tenido en común la creación de mecanismos de acceso al empleo por medio de visas o permisos de trabajo temporales para trabajadores extranjeros. El tema del acceso a la condición de residente permanente - requisito para poder acceder a la opción de ciudadanía por la vía de la naturalización- no ha sido la piedra angular de las propuestas de reforma. De la misma forma, la mayoría de las propuestas de los últimos años enfatiza el sostenimiento del régimen de control y castigo establecido por la ley de inmigración de 1996. Estos énfasis en lo referente al tipo de reforma a la política de inmigración que se visualiza no son planteamientos exclusivos de un partido, por el contrario, expresan el punto de convergencia bipartidista.

El riesgo de una reforma a la ley de inmigración que mantenga e incluso fortalezca la lógica del control, la exclusión, la restricción y el castigo a las comunidades mi- 
grantes, y que a su vez otorgue visas temporales de empleo, implica que una amplia gama de la población extranjera — es decir, millones de personas - viviría en condiciones de vulnerabilidad extrema, esto en términos de explotación laboral, además de que estaría sujeta a un régimen altamente punitivo que la haría también muy vulnerable a detenciones y deportaciones continuas. En el mejor de los casos, se trata de una opción que crearía una masa numerosa de personas viviendo en una condición de segunda categoría en la sociedad, sin acceso a derechos económicos, sociales y políticos disponibles a los ciudadanos estadounidenses; en otras palabras, este grupo sufriría de una condición de apartheid económico-social.

Empero, desde la perspectiva de los millones de extranjeros que viven en el país con el constante temor de ser detenidos, arrestados y deportados por carecer de autorización migratoria alguna, la idea de contar al menos con un permiso de trabajo no deja de ser atractiva, pues es vista como una mejoría en relación con la condición actual de total vulnerabi- lidad. Esta situación hace muy difícil conciliar lo que las comunidades inmigrantes quieren -incluso lo que merecen - con lo que puede ser conseguible en el plazo inmediato.

Además de lo anterior, es previsible que el Partido Republicano intente tomar liderazgo en la resolución legislativa del caso de los jóvenes migrantes que residen en Estados Unidos sin autorización migratoria. Consistente con lo anterior, los senadores republicanos Hutchinson, de Texas, McCain y Kyl, de Arizona, presentaron una propuesta de ley ante dicha cámara, relacionada con el tema de los jóvenes migrantes sin papeles. En esencia, dicha propuesta de ley otorgaría visas temporales a los beneficiarios, sin garantía de acceso eventual a la condición de residencia permanente ni ciudadanía.

De igual manera, la bancada republicana de la Casa de Representantes aprobó el pasado 30 de noviembre una propuesta de reforma a la política de inmigración relacionada con trabajadores altamente calificados. La propuesta consiste en la eliminación de 55 mil visas 
anuales actualmente disponibles como parte del programa de visas para la diversidad, conocida popularmente como «la lotería de visas», y su transferencia a las categorías de visas para trabajadores altamente calificados. En adición, la propuesta aprobada con el apoyo casi exclusivo de legisladores republicanos ofrecería un grado de alivio para el caso de familiares de residentes permanentes y ciudadanos de Estados Unidos que están restringidos para reingresar al país debido a las prohibiciones de reingreso de tres y diez años estipuladas en la ley de 1996.

Por el lado demócrata, el senador Chuck Schummer, de Nueva York, ha expresado el deseo del Partido Demócrata de revivir propuestas de reforma a la política de inmigración presentadas a lo largo de los últimos años, las cuales son consistentes con las propuestas de origen republicano. Desdichadamente, el Partido Demócrata no ha sido capaz de producir una nueva narrativa ni un nuevo paradigma político-legislativo sobre cómo resolver de la manera más funcional, humanista, justa y visionaria el di- lema de qué hacer con la población inmigrante que ya reside en el país; tampoco en lo referente a cómo reorientar la política de inmigración hacia el futuro.

Uno de los más grandes obstáculos a la gestión republicana sobre el tema migratorio es la naturaleza de la bancada republicana en el Congreso, tanto en la Casa de Representantes como en el Senado federales. Dentro de la Casa de Representantes, seguirá dominando el ala más conservadora del Partido Republicano, influida por el Tea Party. Dado que sigue habiendo un grupo de representantes demócratas provenientes de distritos congresionales con fuerte influencia republicana, es muy improbable prever que la Casa de Representantes vaya a moverse en la dirección de favorecer una reforma amplia y generosa en lo relativo a la política de inmigración.

En el Senado, se ha visto fortalecido el grupo de senadores republicanos muy comprometidos con el Tea Party. Esta situación hace difícil prever la posibilidad de conseguir el apoyo de cuando menos cinco senadores republicanos dispuestos a apo- 
yar reformas que vayan más allá de los parámetros antes mencionados. E1 senador republicano de Florida, Marco Rubio, se ha posicionado ya sobre el tema migratorio, enfatizando el favorecimiento de los esquemas de visas temporales o permisos de trabajo, excluyendo el acceso a la condición de residencia permanente. El caso del senador republicano de Carolina del Sur, Lindsey Graham, será interesante de observar, ya que ha sido parte del grupo pragmático del Partido Republicano sobre el tema de política de inmigración; sin embargo, seguramente enfrentará desafíos en las primarias republicanas, provenientes del ala más conservadora del partido en la próxima elección del año 2014, lo cual pudiera resultar en una neutralización efectiva de su liderazgo.

En conclusión, es obvio que a pesar del resultado electoral del pasado 6 de noviembre y del resurgimiento del tema de reforma a la política de inmigración posterior a las elecciones, las dinámicas políticas y electorales locales seguramente ejercerán un peso importante en la manera en que el tema será abordado. La única posibilidad de encausar el tema de la reforma a la política de inmigración en una dirección positiva será a partir de un fuerte trabajo de educación pública e incidencia política que ilustre de manera clara y precisa su naturaleza deshumanizante y obsoleta. El grado de organización de las comunidades migrantes mismas será decisivo para mantener un enfoque de largo plazo sobre este campo de la política pública. Independientemente de qué sea posible conseguir en el corto plazo, será crucial mantener claridad y disciplina sobre las metas de largo plazo. 